\title{
La obesidad abdominal aumenta el riesgo de pólipos colorrectales
}

\author{
Abdominal Obesity Increases the Risks of Colorectal Polyps
}

Óscar Fernando Ruiz Morales, MD, ${ }^{1}$ William Otero Regino, MD, ${ }^{2}$ Martín Alonso Gómez Zuleta, MD, ${ }^{3}$ Dennys Castro Soteldo, MD. ${ }^{4}$

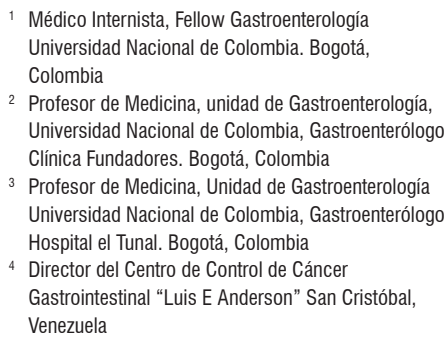

Médico Internista, Fellow Gastroenterología Universidad Nacional de Colombia. Bogotá, Colombia

2 Profesor de Medicina, unidad de Gastroenterología, Universidad Nacional de Colombia, Gastroenterólogo Clínica Fundadores. Bogotá, Colombia

3 Profesor de Medicina, Unidad de Gastroenterología Universidad Nacional de Colombia, Gastroenterólogo Hospital el Tunal. Bogotá, Colombia

${ }^{4}$ Director del Centro de Control de Cáncer Gastrointestinal "Luis E Anderson" San Cristóbal, Venezuela

Fecha recibido: $\quad 06-05-14$ Fecha aceptado: 05-11-14

\begin{abstract}
Resumen
Introducción: recientemente varias publicaciones internacionales han encontrado asociación entre obesidad y pólipos y/o cánceres colorrectales. En nuestro país no se ha estudiado esta posible asociación. El objetivo del presente estudio fue determinar si los pacientes con obesidad tienen más frecuentemente pólipos 0 cánceres colorrectales. Materiales y métodos: estudio transversal, prospectivo realizado entre marzo de 2012 y junio de 2013 con pacientes remitidos a colonoscopia total por tamización. Participaron en el estudio tres instituciones universitarias: Clínica Fundadores y Hospital El Tunal de Colombia y el Centro de Control de Cáncer Gastrointestinal "Luis E Anderson" de Venezuela. A todos los pacientes se les determinaron el peso, la talla, índice de masa corporal $\left(\mathrm{kg} / \mathrm{m}^{2}\right)$ y el perímetro abdominal en centímetros. Se estimó la prevalencia de pólipos en la población y se determinó el riesgo mediante OR con sus intervalos de confianza (IC) de 95\%. Resultados: se incluyeron 405 pacientes, 68,9\% eran mujeres, edad promedio 56,1 años +/- 12,9 años, 154 (38\%) presentaron pólipos, de estos $113(73 \%)(p=0,01)$ cumplían con criterios de obesidad abdominal y 41 $(27 \%)(p=0,03)$, no cumplían con estos criterios. El análisis de regresión logística encontró relación entre el aumento de perímetro abdominal y presencia de pólipos en el colon y por cada cm de aumento del perímetro abdominal se aumenta el riesgo de padecer pólipos en 5,3\%. Conclusión: en la población examinada se encontró que los pacientes con obesidad, más frecuentemente tienen pólipos del colon y este riesgo aumenta con la severidad de la obesidad.
\end{abstract}

\section{Palabras clave}

Obesidad, sobrepeso, pólipos, cáncer.

\begin{abstract}
Introduction: Recently several international publications have found an association between obesity and polyps and/or colorectal cancers. In our country the possibilities of these associations have not been studied. The aim of this study was to determine whether obese patients have polyps or colorectal cancers more frequently than found in the general population. Materials and Methods: This was a cross-sectional prospective study of patients referred for total colonoscopies for screening that were conducted between March 2012 and June 2013. Three university hospitals, Clínica Fundadores and Hospital El Tunal in Bogotà, Colombia and the Centro de Control de Cancer Gastrointestinal "Luis E Anderson" in Venezuela, participated in the study. All patients were weighed and height, body mass index $\left(\mathrm{kg} / \mathrm{m}^{2}\right)$ and waist circumference in centimeters were measured. The prevalence of polyps in the population was estimated and the risk was determined by means of Odds Ratios (OR) with 95\% confidence intervals $(\mathrm{Cl})$. Results: 405 patients were included in the study, $68.9 \%$ of whom were women. The mean patient age was 56.1 years $+/-12.9$ years. $154(38 \%)$ had polyps, of these $113(73 \%)(p=0.01)$, met the criteria for abdominal obesity, and $41(27 \%)(p=0.03)$ did not meet these criteria. Logistic regression analysis found no relationship between increased abdominal circumference and the presence of polyps in the colon, but for every additional centimeter of waist circumference the risk of polyps increased 5.3\%. Conclusion: In the population examined, obese patients had polyps more frequently than did others, and this risk increased with the severity of obesity.
\end{abstract}

\section{Keywords}

Obesity, overweight, polyps, cancer. 


\section{INTRODUCCIÓN}

La obesidad se asocia con un gran número de enfermedades, incluyendo diabetes mellitus tipo 2, hipertensión arterial, enfermedades cardiovasculares, e incluso algunos tipos de cáncer (1) y a nivel mundial, se está produciendo un aumento de su prevalencia (1-3). En el 2007, 523 millones personas en el mundo y más de $30 \%$ de la población americana eran obesas (IMC > 30) (2), en 2008, 1.400 millones de adultos (mayores de 20 años) tenían sobrepeso (2). En 2010, 40 millones de niños (< 5 años), tenían sobrepeso (3) y en Colombia, para 2007, el promedio de personas adultas con sobrepeso fue de $46,02 \%$ y obesidad de $13,71 \%$ (4). Existe evidencia creciente sobre los riesgos para la salud relacionados con la obesidad no solo con la cantidad de grasa corporal total, sino también con la distribución de la grasa corporal en la región visceral (5), por lo cual la medida de la circunferencia de la cintura es un predictor útil e independiente para definir el riesgo cardiovascular, así como también el riesgo de los cánceres secundarios a la obesidad $(6,7)$. Esta adiposidad visceral es un determinante importante de la resistencia a la insulina, la cual es considerada un trastorno temprano y fundamental para el desarrollo de las enfermedades relacionadas con la obesidad (5). En la última década, múltiples estudios epidemiológicos han encontrado un mayor riesgo para el desarrollo de adenomas colorrectales $(5,7-13)$ y cáncer de colon (CC) (13-15) con el aumento de la relación cintura / cadera y/o de la circunferencia de la cintura. Los pólipos adenomatosos son los precursores de la mayoría de los cánceres colorrectales esporádicos (secuencia adenomacarcinoma) (16) y el papel de los adenomas en el desarrollo del cáncer colorrectal (CCR) se estableció cuando Vogelstein y cols demostraron que la acumulación gradual de alteraciones moleculares como mutaciones en la vía APC, seguida por mutaciones en KRAS y p53 determinan la transformación de los pólipos adenomatosos pequeños en pólipos de mayor tamaño con displasia y finalmente de carcinomas invasivos (17). Esta secuencia es la base de la implementación de tamización con el fin de detectar y extirpar oportunamente los pólipos para prevenir el desarrollo de CCR $(10,18)$. El CCR es la tercera causa de muerte por cáncer en EE.UU (20) y la supervivencia a cinco años es del $90 \%$ cuando se detecta en estadio I y menos de $5 \%$ cuando está en estadio IV (19-21). En Colombia, el CCR es la cuarta causa de muerte por cáncer para ambos géneros (22). Su aparición está asociada tanto a factores genéticos como ambientales $(1,19)$ y dentro de estos se destacan el consumo de tabaco y alcohol, una actividad física escasa, algunos patrones dietéticos (bajo consumo de fruta y verdura, mayor contenido en grasa) y la obesidad (23). Esta última aumenta el riesgo de mortalidad por cualquier causa en 30\%, para las enfermedades cardiovasculares en $40 \%$, y para todos los tipos de cáncer en 10\% (24). Así, no solo puede influir en la incidencia de cáncer, sino también afecta el pronóstico, la supervivencia global y la supervivencia libre de enfermedad (25). No obstante este conocimiento, tanto en Colombia como en Suramérica, no se han realizado estudios epidemiológicos sobre la asociación entre obesidad y presencia de pólipos colónicos (adenomas), por lo cual se decidió realizar el presente trabajo.

\section{MATERIALES Y MÉTODOS}

Es un estudio observacional analítico, de tipo transversal en el que se tomó la información de manera prospectiva entre marzo de 2012 y junio de 2013. El tamaño de la muestra se calculó teniendo en cuenta los siguientes criterios: la prevalencia de pólipos esperada en la población general, la cual se encontró tan alta como $25 \%$ a los 50 años de edad (26, $27,29)$ y $50 \%$ a los 70 años $(28,29)$. De manera global, la prevalencia de pólipos colorrectales se ha estimado en 20-60\% en la población occidental; según estudios basados en autopsias (30), en Colombia no se conocen datos de prevalencia de pólipos colónicos por lo que decidimos realizar el cálculo del tamaño de muestra basados en una prevalencia media de $30 \%$, se utilizó una confiabilidad de $95 \%$, poder estadístico de $80 \%$, obteniendo así un tamaño de muestra de 363 pacientes. Se incluyeron todos los pacientes mayores de 18 años a los cuales, previa firma de consentimiento informado, les fue realizada una colonoscopia total diagnóstica catalogada como de calidad teniendo en cuenta los siguientes aspectos: limpieza evaluada por la escala de Boston $(30,31,35)$, con un puntaje mayor a 2 en cada uno de los tres segmentos evaluados (recto-ángulo esplénico, ángulo esplénico-ángulo hepático, ángulo hepático-ciego), con total visualización de los segmentos de colon (observación endoscópica de ciego, válvula ileocecal y agujero apendicular), tiempo de retirada mínimo de seis minutos (6-10 minutos). Con el fin de tener un procedimiento endoscópico confiable, estos fueron realizados por gastroenterólogos expertos (WOR, MAGZ, DCS) docentes de gastroenterología que cumplen con altos estándares de calidad en estos procedimientos (32-35) y cada uno con más 10.000 colonoscopias realizadas. Se excluyeron los pacientes con cáncer de colon conocidos, antecedentes de resección previa de pólipos colónicos, colitis de cualquier etiología y/o cirugía previa de colon. A todos los pacientes se les aplicó un cuestionario con las siguientes variables: edad (años cumplidos), género, antecedentes personales de hipertensión arterial, diabetes mellitus tipo 2, dislipidemia y antecedentes familiares de cáncer de colon en familiares de primer grado. Inmediatamente antes de la realización de la colonoscopia, personal de enfermería 
entrenado para este propósito midió la talla (metros), circunferencia de la cintura $(\mathrm{cm})$ (tomada en el punto medio entre el borde inferior de la última costilla y la cresta ilíaca en un plano horizontal, por encima de las crestas ilíacas, mediciones alrededor de torso desnudo, después de que el paciente exhala mientras está de pie sin zapatos, y los brazos colgando libremente, según ha sido descrito y recomendado previamente) (38-41), peso ( $\mathrm{kg}$ ) sin ropa, índice de masa corporal (IMC) se calculó como el peso $(\mathrm{kg})$ dividido por la altura al cuadrado $\left(\mathrm{m}^{2}\right)$. La relación entre peso y talla, se hizo con base en las definiciones de la Organización Mundial de la Salud (OMS): sobrepeso cuando el IMC está entre 25,0 y $29,9 \mathrm{~kg} / \mathrm{m}^{2}$, y obesidad cuando IMC $>30,0 \mathrm{~kg} / \mathrm{m}^{2}(37)$. La colonoscopia se realizó en la forma usual, utilizando preparación con polietilenglicol y electrolitos, en dosis divididas previamente recomendadas (41-48). A todos se les inició la colonoscopia sin sedación y se utilizaba sedación cuando no era tolerada (sedación selectiva) o cuando el médico remitente la solicitaba. Cuando se hacía de esta manera, la sedación siempre era administrada por un anestesiólogo utilizando propofol y remifentanil, cuyas dosis eran elegidas por este especialista de acuerdo a las características individuales de cada paciente. En los pacientes a quienes se administraba sedación, se les canalizaba una vena en el antebrazo derecho, monitoreo constante de los signos vitales y la saturación de oxígeno y el procedimiento era asistido por al menos 2 enfermeras con entrenamiento en endoscopia digestiva. La recolección de los datos de la población fueron incluidos en tablas de datos virtuales de "Google drive" (URL goo. $\mathrm{gl} / 7 \mathrm{j} 181$ ). Los pacientes elegibles eran seleccionados antes de cada colonoscopia por residentes de medicina interna o por "fellows" de gastroenterología o por asistentes de gastroenterología entrenados y familiarizados con el protocolo de esta investigación. Ellos consignaban inicialmente los datos demográficos y los concernientes a factores de riesgo de cada paciente y posteriormente los resultados de la colonoscopia. El endoscopista no diligenciaba los formularios y no siempre sabía qué pacientes estaban haciendo parte del estudio. Personas ajenas a las endoscopias, semanalmente "bajaban de la nube virtual" los datos de cada paciente y los digitaban en las respectivas bases de datos de la investigación para su análisis posterior.

\section{ANÁLISIS ESTADÍSTICO}

Se tomó como variable dependiente la presencia de pólipos en colonoscopia y como variables independientes: género, edad, antecedentes clínicos (hipertensión arterial, diabetes mellitus, dislipidemia, antecedente de infarto agudo de miocardio y/o enfermedad cerebrovascular). Inicialmente se realizó una descripción de la población para las variables independientes y dependientes utilizando tablas de distribución de frecuencias y para la edad la media aritmética y la desviación estándar (tabla 1), posteriormente se realizaron pruebas exploratorias de datos (chi cuadrado, $t$ de student, prueba Nova), utilizando el programa "IBM SPSS Statistics versión 21.0", calculando el odds ratio (OR) y sus intervalos de confianza (IC 95\%) (tabla 2); por último y con el fin de controlar simultáneamente el efecto sobre la variable dependiente de todas las variables independientes se construyó un modelo de regresión logística.

\section{RESULTADOS}

Durante el periodo del estudio (marzo de 2012 a junio de 2013), se incluyeron 405 formularios de igual número de pacientes sometidos a colonoscopia total diagnóstica que cumplían con los criterios de inclusión, 272 Clínica Fundadores (Bogotá, Colombia), 84 Hospital El Tunal (Bogotá, Colombia) y 49 del centro de control del cáncer (San Cristóbal, Venezuela), de los cuales 279 (68,9\% ) fueron mujeres y $126(31,1 \%)$ hombres con una edad promedio de 56,1 años +/- 12,9 años, de los cuales 185 (45,7\%) tenían antecedentes personales positivos para hipertensión arterial, diabetes mellitus, dislipidemia, infarto agudo de miocardio y/o enfermedad cerebrovascular, 43 (10,6\%) tenían antecedentes familiares de cáncer de colon en primera línea de consanguinidad, 170 (41,9\%) tenían un índice de masa corporal (IMC) normal, 182 (44,9\%) estaban en rango de sobrepeso (IMC $25-29,9 \mathrm{~kg} / \mathrm{m}^{2}$ ) y 53 $(13 \%)$ eran obesos $\left(\right.$ IMC $\left.>30 \mathrm{~kg} / \mathrm{m}^{2}\right), 225(55,5 \%)$ cumplían con criterios de obesidad abdominal (International Diabetes Federation), 154 (38\%) presentaron pólipos durante la colonoscopia (tabla 1), de los cuales 113 (73\%) ( $\mathrm{P}=0.01)$, cumplían con criterios de obesidad abdominal y $41(27 \%)(\mathrm{p}=0,03)$, no cumplían con criterios de obesidad abdominal; con OR de 3,42 con IC 95\% 2,21-5,29), para la asociación entre la presencia de pólipos y obesidad abdominal lo cual es estadísticamente significativo (figura 1); del $55,5 \%(n=225)$, de los pacientes con obesidad abdominal $44,4 \%(n=100)$ presentaron pólipos, en comparación con la población sin criterios de obesidad abdominal que fue de $44,5 \%(n=180)$, de los cuales solo $30 \%(n=54)$, presentó pólipos.

Posteriormente, al realizar las pruebas exploratorias en análisis univariado se encontró que para las variables independientes de género (OR 1,055; IC 95\% 0,684-1,625; P= 0,810 ) y talla (OR 0,305; IC 95\% 0,025-3,722: $\mathrm{P}=0,352$ ) no hubo significancia, lo que indica la ausencia de asociación entre estas variables y la presencia de pólipos en la colonoscopia diagnóstica. 
Para las variables independientes de antecedentes familiares de cáncer de colon en primer grado de consanguinidad (OR 2,254; IC 95\% 1,190-4,271; P=0,013), antecedentes personales positivos para hipertensión arterial, diabetes mellitus, dislipidemia, infarto agudo de miocardio y/o enfermedad cerebrovascular (OR 1,63; IC 95\% 1,092$2,452 ; \mathrm{P}=0,017)$, índice de masa corporal (IMC) (OR 1,131; IC 95\% 1,073-1,193; $\mathrm{P}=0,000$ ), perímetro abdominal (OR 1,053; IC 95\% 1,034-1,071; P=0,000), peso (OR 1,032; IC 95\% 1,014-1,051; P=0,000), edad (OR 1,024; IC 95\% 1,008-1,040; $\mathrm{P}=0,004$ ) (tabla 2), se encontró una significancia estadística de leve a moderada en orden descendente, lo que indica la presencia de asociación entre la presencia de pólipos en la colonoscopia diagnóstica y estas; por ello, se llevaron a un análisis de regresión logística con el fin de explorar las variables que pudiesen contribuir a la explicación de la aparición de pólipos en la colonoscopia diagnóstica, se examinaron los efectos principales de cada una de las variables ajustando simultáneamente a todas las demás consideradas en el modelo, analizando las dos pruebas de bondad de ajuste que muestran que no hay diferencias entre el modelo logístico y los datos observados (tabla 3), encontrando que el perímetro abdominal fue la única variable que ajustada simultáneamente a todas las demás variables tenía significancia estadística, lo que indica la presencia de asociación entre la presencia de pólipos y el aumento en el perímetro abdominal (OR 1,053; IC 95\% 1,034-1,071; $\mathrm{P}=0,000)$.

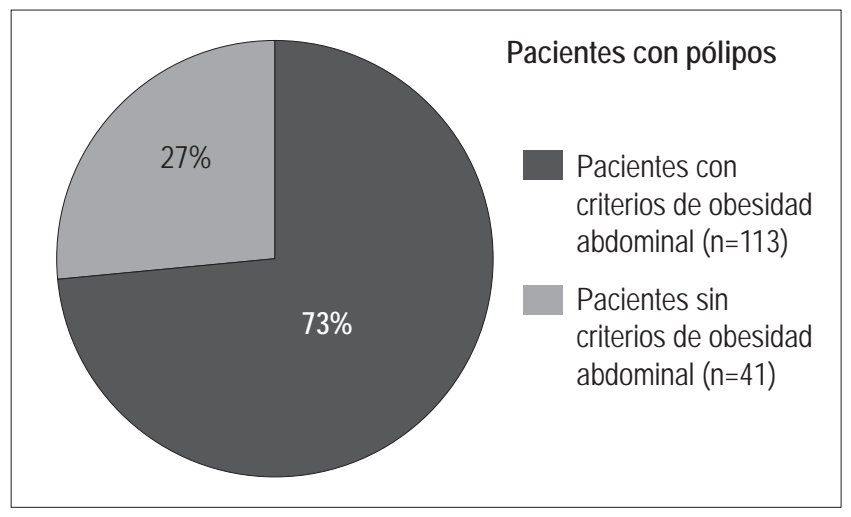

Figura 1. Prevalencia de pólipos de acuerdo a la obesidad.

Se realizó un nuevo modelo de regresión logística que cumplía con características similares respecto al inicial, sin la variable peso, ya que está inmersa en la definición del índice de masa corporal la cual fue llamada simple encontrado el mismo comportamiento de las variables en donde existió significancia estadística para la variable perímetro abdominal (tabla 4).
Tabla 1. Características de los pacientes.

\begin{tabular}{|c|c|}
\hline & $\%(n)$ \\
\hline \multicolumn{2}{|l|}{ Género } \\
\hline Femenino & $689(279)$ \\
\hline Masculino & $31,1(126)$ \\
\hline Edad promedio (años) & 56,1 (Ds 12,9) \\
\hline \multicolumn{2}{|c|}{ Antecedentes personales* } \\
\hline $\mathrm{Si}$ & $45,7(185)$ \\
\hline No & $54,3(220)$ \\
\hline \multicolumn{2}{|c|}{ Antecedente familiar de cáncer de colon } \\
\hline Si $\quad$ & $10,6(43)$ \\
\hline No & $89,4(362)$ \\
\hline \multicolumn{2}{|l|}{ IMC $\left(\mathrm{kg} / \mathrm{m}^{2}\right)$} \\
\hline$<19,9$ & $6,6(27)$ \\
\hline $20-24,9$ & $35,3(143)$ \\
\hline $25-29,9$ & $44,9(182)$ \\
\hline $30-34,9$ & $10,1(41)$ \\
\hline$>35$ & $2,9(12)$ \\
\hline \multicolumn{2}{|c|}{ Perímetro abdominal (cm) } \\
\hline Hombres $>94$ & $13,3(54)$ \\
\hline Mujeres $>80$ & $42,2(171)$ \\
\hline Total & $55,5(225)$ \\
\hline \multicolumn{2}{|c|}{ Presencia de pólipos en la colonoscopia } \\
\hline Femenino & $37,6(105)$ \\
\hline Masculino & $38,8(49)$ \\
\hline Total & $38(154)$ \\
\hline
\end{tabular}

Ds desviación estándar

*Antecedentes de hipertensión arterial, dislipidemia, diabetes mellitus, infarto agudo de miocardio y/o enfermedad cerebrovascular; las frecuencias se hallaron teniendo en cuenta el total de pacientes.

Tabla 2. Análisis estadístico exploratorio univariado.

\begin{tabular}{lllcc}
\hline $\begin{array}{c}\text { Variable } \\
\text { dependiente }\end{array}$ & \multicolumn{1}{c}{$\begin{array}{c}\text { Variable } \\
\text { independiente }\end{array}$} & $\begin{array}{c}\text { Odds Ratio } \\
\text { (OR) }\end{array}$ & $\begin{array}{c}\text { Intervalo de } \\
\text { confianza } \\
95 \%\end{array}$ & $\begin{array}{c}\mathbf{P} \\
(<0,05)\end{array}$ \\
\hline $\begin{array}{l}\text { Presencia de } \\
\text { pólipos }\end{array}$ & Género & 1,055 & $0,684-1,625$ & 0,810 \\
& Edad & 1,024 & $1,008-1,040$ & 0,004 \\
& $\begin{array}{l}\text { Antecedente } \\
\text { clínicos }\end{array}$ & 1,63 & $1,092-2,452$ & 0,017 \\
& $\begin{array}{l}\text { Antecedentes } \\
\text { de cáncer de }\end{array}$ & 2,254 & $1,190-4,271$ & 0,013 \\
& colon & & & \\
& Perímetro & 1,053 & $1,034-1,071$ & 0,000 \\
& abdominal & & & \\
& Talla & 0,305 & $0,025-3,722$ & 0,352 \\
& Peso & 1,032 & $1,014-1,051$ & 0,000 \\
& Índice de masa & 1,131 & $1,073-1,193$ & 0,000 \\
& corporal & & & \\
\hline
\end{tabular}


Tabla 3. Análisis de regresión logística saturado.

\begin{tabular}{lccc}
\hline \multicolumn{1}{c}{ Pólipos colónicos } & $\begin{array}{c}\text { Odds Ratio } \\
\text { (OR) }\end{array}$ & $\begin{array}{c}\text { Intervalo de } \\
\text { confianza 95\% }\end{array}$ & $\mathrm{P}(<0,05)$ \\
\hline Antecedente clínicos & 0,902 & $0,554-1,459$ & 0,679 \\
$\begin{array}{l}\text { Antecedentes de cáncer } \\
\text { de colon }\end{array}$ & 0,436 & $0,219-0,867$ & 0,018 \\
Edad & 1,016 & $0,997-1,035$ & 0,105 \\
Perímetro abdominal & 1,045 & $1,021-1,069$ & 0,000 \\
Índice de masa corporal & 1,073 & $0,973-1,183$ & 0,160 \\
Peso & 0,982 & $0,950-1,016$ & 0,982 \\
\hline
\end{tabular}

Tabla 4. Análisis de regresión logística simple.

\begin{tabular}{lccc}
\hline \multicolumn{1}{c}{ Pólipos colónicos } & $\begin{array}{c}\text { Odds } \\
\text { Ratio (OR) }\end{array}$ & $\begin{array}{c}\text { Intervalo de } \\
\text { confianza 95\% }\end{array}$ & $\mathrm{P}(<0,05)$ \\
\hline $\begin{array}{l}\text { Antecedente clínicos } \\
\text { Antecedentes de cáncer }\end{array}$ & 0,923 & $0,568-1,498$ & 0,745 \\
de colon & 0,435 & $0,219-0,865$ & 0,018 \\
Edad & 1,018 & $0,999-1,037$ & 0,61 \\
Perímetro abdominal & 1,042 & $1,019-1,065$ & 0,000 \\
Índice de masa corporal & 1,035 & $0,964-1,113$ & 0,341 \\
\hline
\end{tabular}

\section{DISCUSIÓN}

En el presente estudio, la tasa de detección de pólipos en las colonoscopias de tamización fue de 38\% (mujeres 37,6 , hombres $37,8 \%$ ), la cual es ligeramente superior a lo recomendado en los estándares actuales de calidad en colonoscopia, que estiman una tasa de detección de pólipos superior a $15 \%$ en mujeres y $25 \%$ en hombres (41-47). Se encontró una asociación estadísticamente significativa entre la obesidad abdominal y la presencia de pólipos. De 154 (38\%) pacientes que tenían pólipos, 113 (73\%) cumplían con criterios de obesidad abdominal $(\mathrm{P}=0,01)$. Así mismo, el 55,5\% $(\mathrm{n}=225)$ de los pacientes con obesidad abdominal tenían pólipos versus el 30\% de los pacientes no obesos $(p<0,01)$. En este último grupo, la prevalencia de pólipos fue superior a la tasa esperada de $25 \%$ para pacientes de población general mayores de 55 años (26, $27,29,42-45)$. Con base en esto hallazgos, el análisis de regresión logística tanto simple como saturado confirmó la asociación estadística significativa entre la obesidad abdominal y la prevalencia de pólipos encontrando que por cada centímetro por encima de los límites que definen la obesidad abdominal el riesgo de padecer pólipos aumenta $5,3 \%$ (OR 1,053; IC 95\% 1,034-1,071; P= 0,000). Esta asociación entre la obesidad abdominal y la presencia de pólipos colónicos es similar a la encontrada en otras latitudes. Recientemente, en un estudio con 126 hombres obesos (IMC $>30 \mathrm{~kg} / \mathrm{m}^{2}$ ), sometidos a colonoscopia de tamización (49), se encontró que eran 6,5 veces más propensos a tener tres o más pólipos de colon que los hombres con un índice de masa corporal normal de igual edad y además los que tenían más de $114 \mathrm{~cm}$ de cintura fueron casi 5 veces más propensos a tener tres o más pólipos que los hombres con una circunferencia abdominal más baja (menor a $96 \mathrm{~cm}$ ). En un estudio japonés realizado en 727 mujeres, se encontró que una circunferencia de cintura $>80 \mathrm{~cm}$, se correlacionó con mayor riesgo de tener pólipos colónicos o CCR (50). En Corea también se ha encontrado un mayor riesgo de pólipos cuando la circunferencia de la cintura es $\geq 90 \mathrm{~cm}$, HR de 1,42; IC 95\% 1,06-1,90) por cada centímetro de circunferencia abdominal superior al definido (46). Nuestros resultados también son comparables con otro estudio japonés recientemente publicado (51) en el cual se encontró que por cada $\mathrm{cm}$ por encima del valor normal del índice de circunferencia abdominal aumentó significativamente el riesgo de pólipos en las mujeres (OR 1,3 IC 1,05-1,64 p=0,01) pero no en los hombres del estudio (51). En Estados Unidos también se ha demostrado una fuerte asociaron con el riesgo de adenomas de colon (para el cuartil más alto versus el más bajo: $\mathrm{OR}=2,38$, IC del 95\% 1,45-3,92) (52) y con carcinoma colorrectal. Un metaanálisis de estudios de cohortes (53) encontró que en comparación al cuartil más bajo versus el cuartil más alto de la circunferencia de la cintura, el RR combinado para el cáncer de colon fue de 1,68 IC 95\%: 1,36-2,08 para los hombres y 1,48 IC 95\%: 1,19-1,84 para las mujeres. La plausibilidad biológica de esta asociación no es clara en el momento actual; sin embargo, algunas características biológicas del adipocito pueden ser tenidas en cuenta. Estas células altamente diferenciadas, además de sus propiedades metabólicas de almacenamiento (glucosa y de triglicéridos), sintetizan adiponectinas que pueden inducir proinflamación $(1,54-56)$ y además proliferación celular por la vía del factor de crecimiento derivado de la insulina $(1,56,57)$. La leptina, otro producto de esas células se ha relacionado con el desarrollo de tumores (58). Además de su actividad proangiogénica, la leptina puede aumentar la tasa de crecimiento de las células endoteliales y suprimir la apoptosis a través de un mecanismo dependiente de $\mathrm{Bcl}-2$ y puede actuar como un factor mitógeno, causando transformación y migración para muchos tipos celulares diferentes (59, $60)$. En conclusión, en este trabajo se encontró una asociación estadísticamente significativa entre la obesidad abdominal representada por la circunferencia de la cintura y la presencia de pólipos colónicos en la población estudiada. Esta asociación también ha sido encontrada en estudios de diferentes países. Teniendo en cuenta la repercusión de la obesidad sobre la mucosa colónica, se debería enviar a los pacientes obesos a colonoscopia de tamización antes de los 50 años de edad, aunque se necesitarían más estudios para determinar el impacto de esta recomendación. 


\section{Conflicto de intereses}

Ninguno. Los costos de la presente investigación fueron asumidos en su totalidad por los investigadores.

\section{REFERENCIAS}

1. Guffey CR, Fan D, Singh UP, et al. Linking obesity to colorectal cancer: recent insights into plausible biological mechanisms. Curr Opin Clin Nutr Metab Care 2013; 16: 595-600.

2. James WP. The epidemiology of obesity: the size of the problem. J Intern Med 2008; 4: 336-352.

3. Burke CA. Colonic complications of obesity. Gastroenterol Clin North Am 2010; 1: 47-55.

4. Rodríguez J, Ruiz F, Peñaloza E, et al. Encuesta Nacional de Salud 2007. Resultados nacionales. Bogotá, Colombia: Ministerio de la Protección Social; 2009.

5. Kim Y, Kim Y, Lee S. An association between colonic adenoma and abdominal obesity: a cross-sectional study. BMC Gastroenterol 2009; 9: 4-10.

6. Yamaji T, Iwasaki M, Sasazuki S, et al. Visceral fat volume and the prevalence of colorectal adenoma. Am J Epidemiol 2009; 170: 1502-11.

7. Nguyen DM, El-Serag HB. The epidemiology of obesity. Gastroenterol Clin North Am 2010; 39: 1-7.

8. Janssen I, Katzmarzyk PT, Ross R. Waist circumference and not body mass index explains obesity-related health risk. Am J Clin Nutr 2004; 79: 379-84.

9. Wise LA, Rosenberg L, Palmer JR, et al. Anthropometric risk factors for colorectal polyps in African-American women. Obesity 2008; 16: 859-868.

10. Liu CS, Hsu HS, Li CI, et al. Central obesity and atherogenic dyslipidemia in metabolic syndrome are associated with increased risk for colorectal adenoma in a Chinese population. BMC Gastroenterol 2010; 27: 10:51.

11. Kim YJ, Lee KM, Chung WC, et al. Association between measures of obesity and colorectal adenoma Chin Med J (Engl) 2011; 124: 3711-5.

12. Otake S, Takeda H, Suzuki Y, et al. Association of visceral fat accumulation and plasma adiponectin with colorectal adenoma: evidence for participation of insulin resistance. Clin Cancer Res 2005; 11: 3642-3646.

13. Yamamoto S, Nakagawa T, Matsushita Y, et al. Visceral fat area and markers of insulin resistance in relation to colorectal neoplasia. Diabetes Care 2010; 33: 184-9.

14. Larsson SC, Rutegård J, Bergkvist L, et al. Physical activity, obesity, and risk of colon and rectal cancer in a cohort of Swedish men. Eur J Cancer 2006; 42: 2590-7.

15. Erarslan E, Turkay C, Koktener A, et al. Association of visceral fat accumulation and adiponectin levels with colorectal neoplasia. Dig Dis Sci 2009; 54: 862-868.

16. MacInnis RJ, English DR, Hopper JL, et al. Body size and composition and colon cancer risk in men. Cancer Epidemiol Biomarkers Prev 2004; 13: 553-559.
17. Vogelstein B, Fearon ER, Hamilton SR, et al. Genetic alterations during colorectal-tumor development. N Engl J Med 1988; 319: 525-32.

18. Bujanda L, Cosme A, Gil I, et al. Malignant colorectal polyps. World J Gastroenterol 2010; 16: 3103-3111.

19. Jemal A, Siegel R, Ward E, et al. Cancer statistics, 2009. CA Cancer J Clin 2009; 59: 225-249.

20. National Cancer Institute. Surveillance Epidemiology and End Results. (2011). Colorectal Cancer Facts and Figures. Revisado julio 25, 2013, desde: http://www. cancer.org/ Research/CancerFactsFigures/ colorectal-cancer-factsfigures-2011-2013.

21. Rivero M, Castro B, Fernández Gil PL. Pólipos y poliposis cólica. Medicine 2012; 11: 431-6

22. Piñeros M, Hernández G, Bray F. Increasing mortality rates of common malignancies in Colombia. Cancer 2004; 101: 2285-92.

23. Perea MD, Castaño-Vinyals G, Altzibar JM, et al. Cancer screening practices and associated lifestyles in population controls of the Spanish multi-case control study. Gac Sanit 2012; 26: 301-10

24. Teucher B, Rohrmann S, Kaaks R. Obesity: focus on allcause mortality and cancer. Maturitas 2010; 65: 112-6.

25. Giovannucci E, Michaud D. The role of obesity and related metabolic disturbances in cancers of the colon, prostate, and pancreas. Gastroenterology 2007; 132: 2208-25.

26. Kristjansdottir S, Jonasson JG, Cariglia N, et al. Colonic adenomas found via colonoscopy: yield and risk factors for high-grade dysplasia. Digestion 2010; 82: 252-7.

27. Soultati A, Alexopoulou A, Dourakis SP, Colonic polyps as incidental findings. European Journal of Internal Medicine 2010; $21: 574-80$.

28. Williams AR, Balasooriya BA, Day DW. Polyps and cancer of the large bowel: a necropsy study in Liverpool. Gut 1982; 23: 835-42.

29. Bacchiddu S, Álvarez-Urturri AC, Bessa Caserras J. Pólipos colorrectales. FMC 2012; 19: 472-80.

30. Calderwood $\mathrm{AH}$, Jacobson $\mathrm{BC}$. Comprehensive validation of the Boston bowel preparation scale. Gastrointest Endosc 2010; 72: 686-92.

31. Lai EJ, Calderwood AH, Doros G, et al. The Boston bowel preparation scale: A valid and reliable instrument for colonoscopy-oriented research. Gastrointest Endosc 2009; 69(3 Pt 2): 620-5.

32. Gonzalez-Huix Llado F, Figa Francesch M, Huertas Nadal C. Essential quality criteria in the indication and performance of colonoscopy. Gastroenterol Hepatol 2010; 33: 33-42.

33. Morán Sánchez S, Torrella E, EP Delgado, et al. Colonoscopy quality assessment. Rev Esp Enferm Dig 2009; 101: 107-12.

34. Jover R, Herráiz M, Alarcón $\mathrm{O}$, et al. Clinical practice Guidelines: quality of colonoscopy in colorectal cancer screening. Endoscopy 2012; 44: 444-51.

35. Lorenzo-Zúñiga V, Moreno de Vega V, Boix J. Preparación para colonoscopia: tipos de productos y escalas de limpieza. Rev Esp enferm dig 2012; 104: 426-431. 
36. Rex DK, Lehman GA, Ulbright TM, et al. Colonic neoplasia in asymptomatic persons with negative fecal occult blood tests: influence of age, gender, and family history. Am J Gastroenterol 1993; 88: 825-31.

37. World Health Organization. Obesity 2012. Disponible en: http://www.who.int/mediacentre/factsheets/ fs311/en/ Acceso julio 12, 2013.

38. Esposito K, Chiodini P, Colao A, et al. Metabolic syndrome and risk of cancer: a systematic review and meta-analysis. Diabetes Care 2012; 35: 2402-11.

39. Alberti KG, Zimmet P, Shaw J, for the IDF Epidemiology Task Force Consensus Group. The metabolic syndrome: a new worldwide definition. Lancet 2005; 366: 1059-72.

40. Alegría Ezquerra E, Castellano Vázquez JM, Alegría Barrero A. Obesity, metabolic syndrome and diabetes: cardiovascular implications and therapy. Rev Esp Cardiol 2008; 61: 752-64.

41. Calderwood AH, Jacobson BC. Colonoscopy Quality Metrics and Implementation. Gastroenterol Clin N Am 2013; 42: 599-618.

42. Schoenfeld PS, Cohen J. MD Quality indicators for colorectal cancer screening for colonoscopy. Techniques in Gastrointestinal Endoscopy 2013; 15: 59-68.

43. Lee RH, Tang RS, Muthusamy VR, et al. Quality of colonoscopy withdrawal technique and variability in adenoma detection rates (with videos). Gastrointest Endosc 2011; 74: 128-34.

44. Lieberman DA, Rex DK, Winawer SJ, et al. Guidelines for colonoscopy surveillance after screening and polypectomy: a consensus update by the US Multi-Society Task Force on Colorectal Cancer. Gastroenterology 2012; 143: 844-57.

45. Klein S, Allison DB, Heymsfield SB, et al. Waist circumference and cardiometabolic risk: a consensus statement from shaping America's health: Association for Weight Management and Obesity Prevention; NAASO, the Obesity Society; the American Society for Nutrition; and the American Diabetes Association. Diabetes Care 2007; 30: $1647-52$.

46. Lorenzo-Zúñiga V, Moreno-de-Vega V, Boix J. Preparation for colonoscopy: types of scales and cleaning products. Rev Esp Enferm Dig 2012; 104: 426-31.

47. Puckett J, Soop M. Optimizing colonoscopy preparation: the role of dosage, timing and diet. Curr Opin Clin Nutr Metab Care 2012; 15: 499-504.
48. Hazewinkel Y, Dekker E. Colonoscopy: basic principles and novel techniques. Nat Rev Gastroenterol Hepatol 2011; 8: 554-64

49. Comstock SS, Hortos K, Kovan B et al. Adipokines and obesity are associated with colorectal polyps in adult males: a cross-sectional study. PLoS One 2014; 17: e85939.

50. Kaneko R, Sato Y, An Y, et al. Clinico-epidemiologic study of the metabolic syndrome and lifestyle factors associated with the risk of colon adenoma and adenocarcinoma. Asian Pac J Cancer Prev 2010; 11: 975-83.

51. Kaneko R, Nakazaki N, Tagawa T et al. A new index of abdominal obesity which effectively predicts risk of colon tumor development in female Japanese. Asian Pac J Cancer Prev 2014; 15: 1005-10.

52. Thompson CL, Berger NA, Chak A, et al. Racial differences in measures of obesity and risk of colon adenoma. Obesity 2012; 20: 673-7.

53. Dai Z, Xu YC, Niu L. Obesity and colorectal cancer risk: a meta-analysis of cohort studies. World J Gastroenterol 2007; 13: 4199-206.

54. Otake S, Takeda H, Suzuki Y, et al. Association of visceral fat accumulation and plasma adiponectin with colorectal adenoma: evidence for participation of insulin resistance. Clin Cancer Res 2005; 11: 3642-3646.

55. Kono S, Handa K, Hayabuchi H, et al. Obesity, weight gain and risk of colon adenomas in Japanese men. Jpn J Cancer Res 1999; 90: 805-811.

56. Schoen RE, Weissfeld JL, Kuller LH, et al. Insulin-like growth factor-I and insulin are associated with the presence and advancement of adenomatous polyps. Gastroenterol 2005; 129: 464-475.

57. Gerson LB. Impact of obesity on endoscopy. Gastrointest Endosc 2009; 70: 758-62.

58. P. Somasundar P. McFadden DW, Hileman SM et al. Leptin is a growth factor in cancer. J Surg Res 2004; 116: 337-349.

59. Drew JE. Molecular mechanisms linking adipokines to obesity-related colon cancer: focus on leptin. Proc Nutr Soc 2012; 71: 175-80.

60. Aleksandrova K, Boeing H, Jenab M, et al. Leptin and soluble leptin receptor in risk of colorectal cancer in the European Prospective Investigation into Cancer and Nutrition cohort. Cancer Res 2012; 72: 5328-37. 